\title{
Percepción sobre seguridad ciudadana en el distrito de Magdalena del Mar, Lima 2018
}

\author{
Perception citizen security in the district of Magdalena del \\ Mar, Lima 2018
}

ID Stalein J. Tamara Tamariz ${ }^{\unrhd}$, iDYrene C. Uribe Hernández², iDNelly N. Tamariz Grados ${ }^{3}$ y (iD) Esteban V. Horna Bances ${ }^{4}$

${ }^{1}$ Universidad Privada del Norte. Lima, Perú

2 Universidad Nacional de Cañete. Lima, Perú

${ }^{3}$ Universidad Nacional José Faustino Sánchez Carrión. Lima, Perú

${ }^{4}$ Universidad Le Cordon Bleu. Lima, Perú

\begin{tabular}{llll}
\hline Recibido: 20/04/2018 & Revisado: 30/04/2019 & Aceptado: 12/05/2019 & Publicado: 30/06/2019 \\
\hline
\end{tabular}

\section{RESUMEN}

Se realizó el presente estudio para determinar los factores relacionados con la empleabilidad de los egresados de la carrera universitaria de gastronomía ofertada por la Universidad Le Cordon Bleu en Lima, Perú. La población estuvo comprendida por 50 profesores, 300 estudiantes de los dos últimos ciclos de la carrera de gastronomía, 216 egresados y 83 potenciales empleadores en la ciudad de Lima. La muestra se determinó al azar: 114 estudiantes y 27 docentes, y egresados y empleadores en una muestra irrestricta.

Se aplicaron encuestas en forma presencial para el caso de los profesores y estudiantes, y virtual para el caso de los egresados y empleadores. Se concluyó que los principales factores fueron: 1) los factores bajo la percepción de los estudiantes son: que siempre le gusta mantener la armonía con sus compañeros, el creer que será un buen profesional si logra obtener un trabajo en su carrera, los antecedentes penales y judiciales, el adaptarse con facilidad a los nuevos cambios tecnológicos, el respeto por la naturaleza, y casi siempre la dependencia que la calidad de un puesto de trabajo tiene de la logística que brinda; 2) los factores bajo la percepción de los egresados fueron siempre la aceptación de su responsabilidad ante un error cometido, el tener un plan organizado para búsqueda de un trabajo, el estado civil, el saber negociar y resolver conflictos, el demostrar afectividad por sus compañeros, y la dependencia que la calidad de un puesto de trabajo tiene de la habilidad del trabajador; 3) los factores bajo la percepción de los docentes fue mayormente bueno en el respeto por el ambiente, manejo de herramientas tecnológicas, tener perfil de líder, ser solidario y mostrar manejo de sus emociones ante situaciones complicadas; 4) los factores bajo la percepción de los empleadores fue mayormente bueno el ser ordenado en sus actividades y el desenvolvimiento en las presentaciones de trabajos encomendados.

En su mayoría los empleadores consideraron que los egresados requerían capacitación y esta capacitación debería ser en habilidades blandas, comportamiento más eficiente en el área de trabajo, idiomas, y capacidad de síntesis y análisis.

Palabras clave: Factores de empleabilidad, gastronomía, percepción. 


\begin{abstract}
The present study was carried out to determine the factors related to the employability of graduates of the university gastronomy career offered by the Le Cordon Bleu University in Lima, Peru. The population was comprised of 50 professors, 300 students from the last two cycles of the gastronomy career, 216 graduates and 83 potential employers in the city of Lima. The sample was determined at random: 114 students and 27 teachers, and graduates and employers in an unrestricted sample. Surveys were applied in person in the case of teachers and students, and virtual in the case of graduates and employers.

It was concluded that the main factors were: 1) The factors under the students' perception are: that you always like to keep harmony with your classmates, believing that you will be a good professional if you get a job in your career, criminal records and judicial, adapting easily to new technological changes, respect for nature, and almost always dependence on the quality of a job has the logistics it provides; 2) The factors under the perception of the graduates were always the acceptance of their responsibility in the face of an error made, having an organized plan to find a job, marital status, knowing how to negotiate and resolve conflicts, demonstrating affection for their peers, and the dependence that the quality of a job has on the skill of the worker; 3) The factors under the teachers' perception were mostly good respect for the environment, management of technological tools, having a leader profile, being supportive and showing management of their emotions in complicated situations; 4) The factors under the employers' perception were mostly good to be ordered in their activities and the performance in the presentations of entrusted work. For the most part employers considered that graduates required training and this training should be in soft skills, more efficient behavior in the area of work, languages, and ability to synthesize and analyze.
\end{abstract}

Keywords: Employability factors, gastronomy, perception.

\section{INTRODUCCIÓN}

En el mundo a diario se observa a través de los noticieros muchos casos de violencia familiar, hacia la mujer, niños, ancianos, sumando a esto aparecen los casos de criminalidad, delincuencia, robos comunes y agravados; lo que antes solo era robo al paso, en estos últimos años los asaltos en su mayoría terminan con heridos o fallecidos no exento de ello puede ser algún pariente, amigo o vecino sin que poco o nada podamos hacer frente a esta barbarie popular que poco a poco nos está dejando aislados de la concurrencia social. Basombrio C. (2003), en su tesis Plan Nacional del Sistema de la Seguridad Ciudadana en lima Perú - 2003, llega a la siguiente conclusión: En nuestro país, según las conclusiones del informe, no existe una política de estado y un sistema de seguridad ciudadana que articula vínculos intersectoriales entre el poder ejecutivo, el poder judicial, las regiones, las municipalidades y la sociedad organizada para hacer frente a la criminalidad y violencia. Cuadra E. (2015), en Nicaragua, a través de una encuesta realizada por el Instituto de Estudios Estratégicos y Políticas Públicas sobre percepciones sobre seguridad ciudadana revelan un punto de inflexión en la percepción ciudadana respecto a la seguridad.

Se mantiene la tendencia de ubicar a la 
seguridad en el tercer o cuarto lugar de importancia entre los principales problemas del país y con porcentajes bastante menores respecto a problemas relacionados con la situación económica.

Esta situación no es ajena a la realidad de nuestro país, donde vivimos una total incertidumbre, a partir de estos casos que se registran en las calles de cada ciudad; tal es así que sin irnos muy lejos el distrito de Magdalena del Mar, somos testigos del incremento de la delincuencia por gente de mal vivir, y como consecuencia el ciudadano de a pie que cuenta con menos recursos económicos y empresarios de la zona que se ven afectados por la inoperatividad de las autoridades de turno, la falta de estrategias, y desinterés del gobierno central en proponer reformas nacionales en el poder judicial, hace que perciban serios indicios de inseguridad en la parte urbana y marginal de esta ciudad.

Por tal motivo, la investigación tuvo como objetivo principal conocer la percepción sobre la seguridad ciudadana por parte de los habitantes del distrito de Magdalena del Mar, Lima.

\section{MATERIALES Y MÉTODOS}

Población: Varones y mujeres que radican en el distrito de Magdalena del Mar.

Muestra: 224 varones y mujeres (entre 18 a 65 años) que radican en el distrito de Magdalena del Mar.

Tipo de investigación: Descriptivo cuantitativo.

Diseño de investigación: No experimental. Metodología: Probabilística.

Instrumento: Cuestionario (validado por 4 expertos y aplicando la prueba de confiabilidad de alfa de Cronbach).

Técnica: Encuesta.

Procesamiento de la información: Se realizó una estadística descriptiva para las preguntas y e intervalos de confianza para estimar las proporciones poblacionales de la variable en estudio. Para el procesamiento de la información se usó el paquete estadístico Excel.

\section{RESULTADOS}

Percepción sobre la inseguridad ciudadana

El principal problema sobre la inseguridad ciudadana son los robos que se producen en el distrito con un $31 \%$.

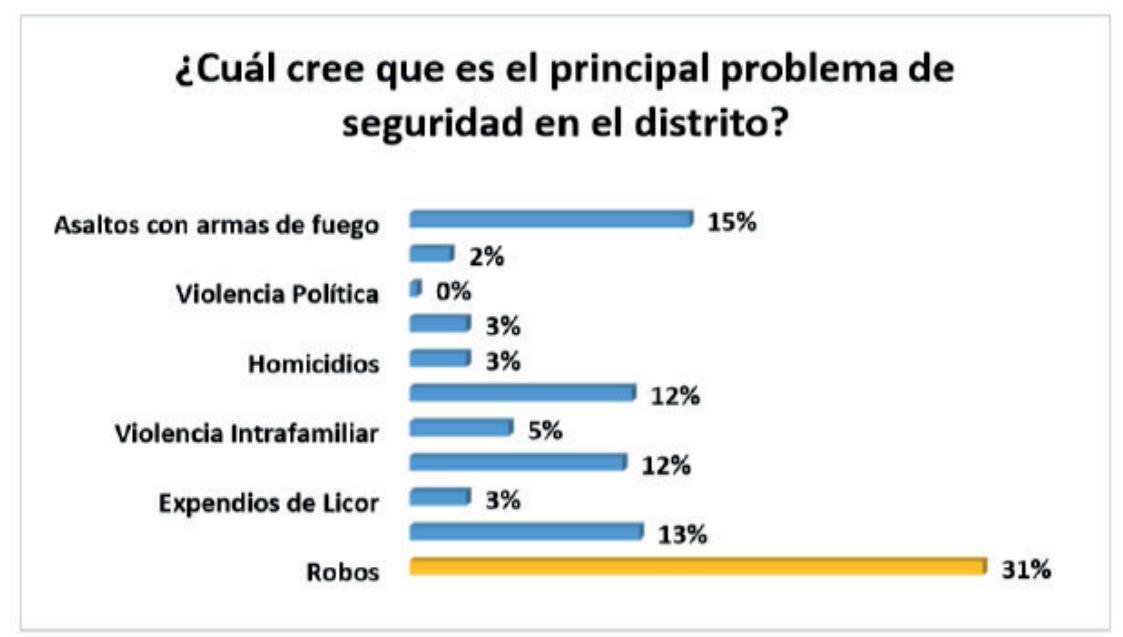

Figura 1. Principal problema sobre seguridad ciudadana. 
La percepción se mantiene igual sobre la seguridad ciudadana en los últimos 12 meses en el distrito de Magdalena del Mar con un 64,70 \%.

\section{En comparación a hace 12 meses, usted diría que en el distrito de Magdalena...}

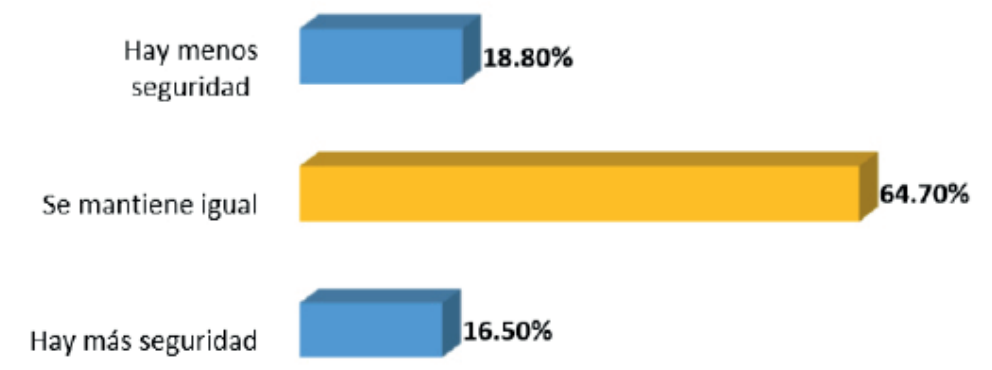

Figura 2. Comparación de la seguridad ciudadana (últimos 12 meses).

La principal causa por la que se produce la inseguridad ciudadana es la falta de policías en el distrito.

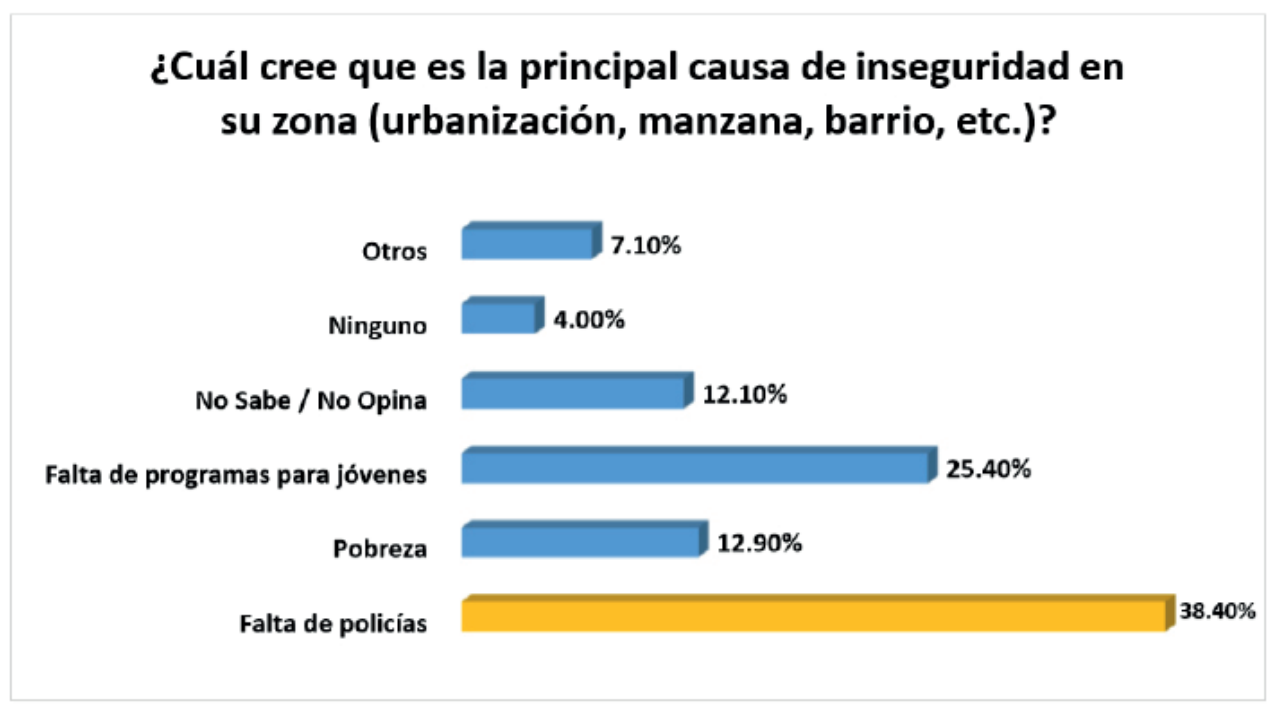

Figura 3. Motivos por el que se produce la inseguridad ciudadana.

El principal delito que ocurre en el distrito de Magdalena del Mar es el robo y tráfico de vehículos: 


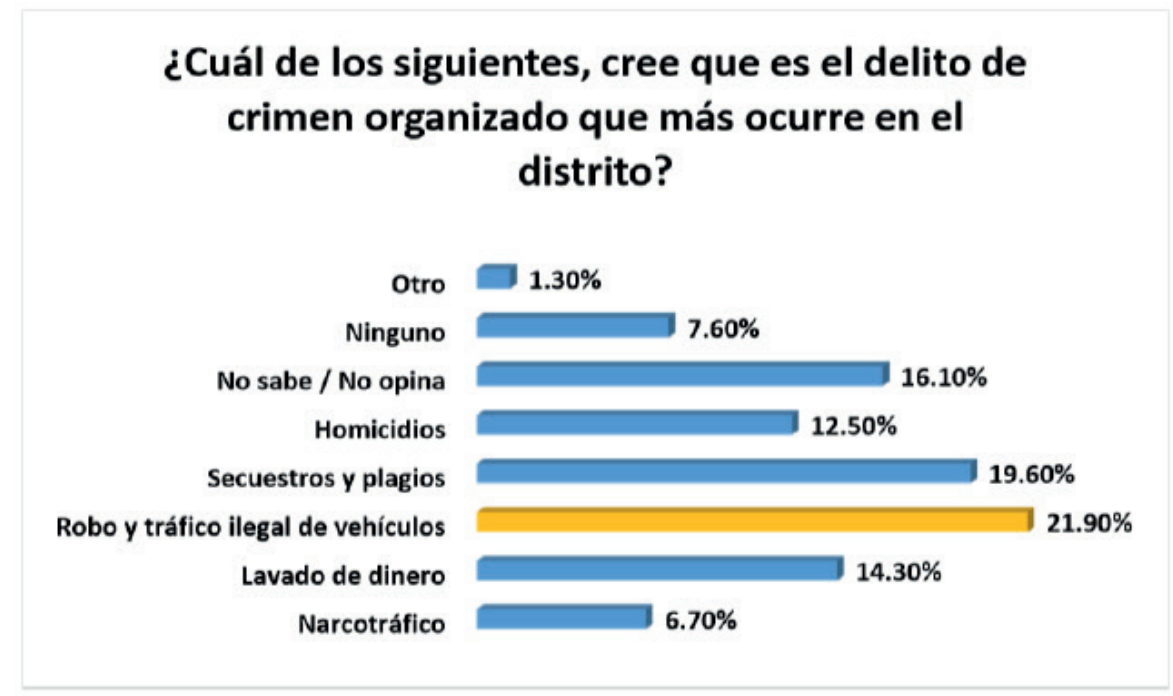

Figura 4. Principal delito de crimen organizado en el distrito.

Uno de los principales problemas sobre el comportamiento individual, en seguridad vial de los habitantes del distrito de Magdalena del Mar, es la falta de respeto a las normas viales.

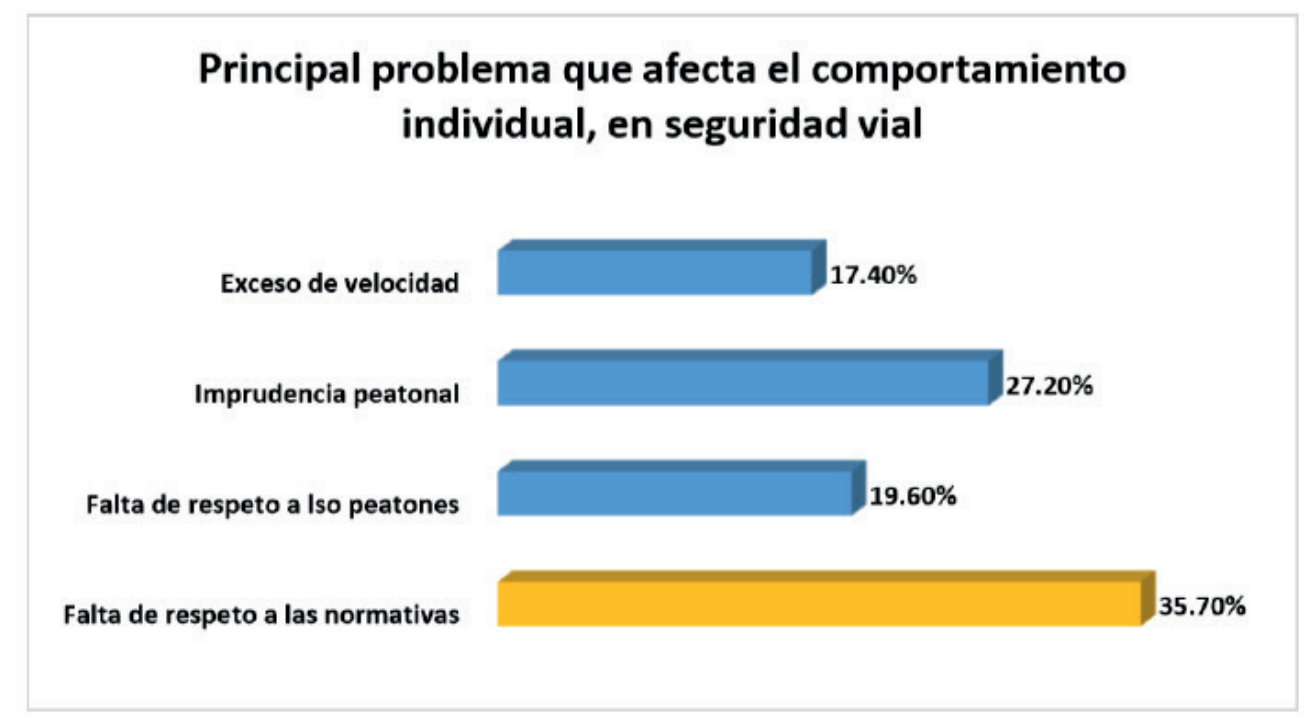

Figura 5. Comportamiento vial. 
Tabla 1. Contrastaciones de las hipótesis

\begin{tabular}{|c|c|c|c|}
\hline \multicolumn{4}{|c|}{$\begin{array}{l}\text { Ho: Menos de un cuarto de los habitantes de Magdalena del Mar afirman que la causa principal de la } \\
\text { inseguridad ciudadana en el distrito es por el robo. }\end{array}$} \\
\hline \multirow{2}{*}{$\begin{array}{l}\text { Grados de } \\
\text { libertad (gl) }\end{array}$} & \multirow{2}{*}{$\begin{array}{l}\text { Significación } \\
\text { bilateral }\end{array}$} & \multicolumn{2}{|c|}{ Intervalo de confianza al $95 \%$} \\
\hline & & Inferior & Superior \\
\hline 223 & 0.000 & $25 \%$ & $37 \%$ \\
\hline \multicolumn{4}{|c|}{ Prueba de hipótesis (95 \% de conflanza) } \\
\hline Muestra & $\begin{array}{l}\text { Muestra } \\
\text { proporcional }\end{array}$ & $\begin{array}{l}\text { Prueba } \\
\text { proporcional }\end{array}$ & Valor de significación \\
\hline 224 & $p>25 \%$ & $\mathrm{p}=31.3 \%$ & 0.015 \\
\hline \multicolumn{4}{|c|}{$\begin{array}{l}\text { Ho: Menos del } 50 \% \text { de los habitantes de Magdalena del Mar ante un acto de inseguridad llaman a la } \\
\text { policía y/o serenazgo. }\end{array}$} \\
\hline \multirow{2}{*}{$\begin{array}{c}\text { Grados de } \\
\text { libertad }(\mathrm{gl})\end{array}$} & \multirow{2}{*}{$\begin{array}{c}\text { Significación } \\
\text { bilateral }\end{array}$} & \multicolumn{2}{|c|}{ Intervalo de confianza al $95 \%$} \\
\hline & & Inferior & Superior \\
\hline 223 & 0.000 & $50 \%$ & $63 \%$ \\
\hline \multicolumn{4}{|c|}{ Prueba de hipótesis (95 \% de confianza) } \\
\hline Muestra & $\begin{array}{l}\text { Muestra } \\
\text { proporcional }\end{array}$ & $\begin{array}{l}\text { Prueba } \\
\text { proporcional }\end{array}$ & Valor de significación \\
\hline 224 & $p>50 \%$ & $p=56.3 \%$ & 0.031 \\
\hline \multicolumn{4}{|c|}{$\begin{array}{l}\text { Ho: Más del } 50 \% \text { de los habitantes de Magdalena del Mar piensa que la policía y serenazgo sean } \\
\text { eficientes a la hora de responder ante un acto de seguridad ciudadana. }\end{array}$} \\
\hline \multirow{2}{*}{$\begin{array}{l}\text { Grados de } \\
\text { libertad (gl) }\end{array}$} & \multirow{2}{*}{$\begin{array}{l}\text { Significación } \\
\text { bilateral }\end{array}$} & \multicolumn{2}{|c|}{ Intervalo de confianza al $95 \%$} \\
\hline & & Inferior & Superior \\
\hline 223 & 0.000 & $32 \%$ & $44 \%$ \\
\hline \multicolumn{4}{|c|}{ Prueba de hipótesis (95 \% de confianza) } \\
\hline Muestra & $\begin{array}{l}\text { Muestra } \\
\text { proporcional }\end{array}$ & $\begin{array}{l}\text { Prueba } \\
\text { proporcional }\end{array}$ & Valor de significación \\
\hline 224 & $P<50 \%$ & $p=37.9 \%$ & 0.000 \\
\hline \multicolumn{4}{|c|}{$\begin{array}{l}\text { Ho: Menos del } 1 / 3 \text { de los habitantes de Magdalena del Mar piensan que la mayor causa de la } \\
\text { inseguridad ciudadana es la falta de policías. }\end{array}$} \\
\hline \multirow{2}{*}{$\begin{array}{c}\text { Grados de } \\
\text { libertad (gl) }\end{array}$} & \multirow{2}{*}{$\begin{array}{l}\text { Significación } \\
\text { bilateral }\end{array}$} & \multicolumn{2}{|c|}{ Intervalo de confianza al $95 \%$} \\
\hline & & Inferior & Superior \\
\hline 223 & 0.000 & $32 \%$ & $45 \%$ \\
\hline \multicolumn{4}{|c|}{ Prueba de hipótesis (95 \% de confianza) } \\
\hline Muestra & Muestra proporcional & $\begin{array}{c}\text { Prueba } \\
\text { proporcional }\end{array}$ & Valor de significación \\
\hline 224 & $\mathrm{p}>33.3 \%$ & $\mathrm{p}=38.4 \%$ & 0.043 \\
\hline \multicolumn{4}{|c|}{$\begin{array}{l}\text { Ho: Menos del } 50 \% \text { de los habitantes de Magdalena del Mar piensan que la seguridad en el distrito de } \\
\text { Magdalena se mantiene igual a comparación de un año atrás. }\end{array}$} \\
\hline \multirow{2}{*}{$\begin{array}{l}\text { Grados de } \\
\text { libertad (gl) }\end{array}$} & \multirow{2}{*}{$\begin{array}{l}\text { Significación } \\
\text { bilateral }\end{array}$} & \multicolumn{2}{|c|}{ Intervalo de confianza al $95 \%$} \\
\hline & & Inferior & Superior \\
\hline 223 & 0.000 & $58 \%$ & $71 \%$ \\
\hline \multicolumn{4}{|c|}{ Prueba de hipótesis (95\% de confianza) } \\
\hline Muestra & Muestra proporcional & $\begin{array}{l}\text { Prueba } \\
\text { proporcional }\end{array}$ & Valor de significación \\
\hline 224 & $p>50.0 \%$ & $p=64.7 \%$ & 0.000 \\
\hline
\end{tabular}


En la tabla 1 podemos observar que:

La proporción de habitantes del distrito de Magdalena de Mar que opina que el robo es la causa principal de la inseguridad ciudadana se encuentra entre el $25 \%$ al $37 \%$.

Y a una significación de $0.05>0.015$ (rechazamos la hipótesis nula) podemos afirmar que más de un cuarto de los habitantes de Magdalena del Mar opina que la causa principal de la inseguridad ciudadana es el robo.

La proporción de habitantes del distrito de Magdalena de Mar ante un acto de inseguridad Ilaman a la policía y/o serenazgo se encuentra entre el $50 \%$ al $63 \%$. Y a una significación de $0.05>0.031$ (rechazamos la hipótesis nula) podemos afirmar que más del $50 \%$ de los habitantes de Magdalena del Mar ante un acto de inseguridad llaman a la policía y/o serenazgo.

La proporción de habitantes del distrito de Magdalena de Mar piensan que la eficiencia de la policía y serenazgo se encuentra entre el $32 \%$ al $44 \%$. Y a una significación de $0,05>0,000$ (rechazamos la hipótesis nula) podemos afirmar que, menos del $50 \%$ de los habitantes de Magdalena del Mar piensa que la policía y serenazgo sean eficientes a la hora de responder ante un acto de seguridad ciudadana.

La proporción de habitantes del distrito de Magdalena de Mar piensan que la inseguridad ciudadana se produce debido a la falta de policía se encuentra entre el $32 \%$ al $45 \%$. Y a una significación de $0.05>0.043$ (rechazamos la hipótesis nula) podemos afirmar que, más del 1/3 de los habitantes de Magdalena del Mar piensan que la mayor causa de la inseguridad ciudadana es la falta de policías.
La proporción de habitantes del distrito de Magdalena de Mar piensan que la seguridad en el distrito de Magdalena del Mar en los últimos 12 meses se mantiene igual se encuentra entre el $58 \%$ al $71 \%$. Y a una significación de $0.05>0.000$ (rechazamos la hipótesis nula) podemos afirmar que, más del $50 \%$ de los habitantes de Magdalena piensan que la seguridad en el distrito de Magdalena se mantiene igual a comparación de un año atrás.

\section{DISCUSIÓN}

Según lo expuesto por Cuadra E. (2015), en Nicaragua a través de una encuesta realizada por Instituto de Estudios Estratégicos y Políticas Públicas sobre percepciones sobre seguridad ciudadana, los resultados revelaron un punto de inflexión en la percepción ciudadana respecto a la seguridad.

Se mantiene la tendencia de ubicar a la seguridad en el tercer o cuarto lugar de importancia entre los principales problemas del país y con porcentajes bastante menores respecto a problemas relacionados con la situación económica.

Esto se corrobora con los resultados obtenidos en el distrito de Magdalena del Mar donde la seguridad ciudadana es uno de los problemas que la población prioriza ante los problemas económicos que pueden tener y que las autoridades deben de asumir como prioridad.

En el caso de la seguridad pública y la percepción de inseguridad, la probabilidad de elaboración supone que los procesos internos son el resultado de estrategias informativas y comunicativas que buscan persuadir a las audiencias mientras las disuaden de sus grupos de referencia para adoptar estilos de vida de grupos delictivos que sustituyan a su 
vida cotidiana (Bolívar, Contreras, Jiménez y Chaux, 2010).

Respecto a la percepción de inseguridad, la disonancia cognitiva es un proceso mental que busca restaurar el orden cognitivo que se desdibujó luego de que los medios de comunicación difundieran noticias en torno a delitos del fuero común o federal que impactaron a las audiencias de un modo tal que se formaron actitudes, se construyeron representaciones y se establecieron habitus discursivos en torno a los cuales el Estado es el responsable directo por su opacidad en el combate a la delincuencia ya que no supo prevenir las causas socioculturales que permitieron el surgimientos de grupos delictivos y su expansión más allá de las fronteras (Araujo, 2012).

La disonancia cognitiva es un concepto que vincula la información general sobre la seguridad pública con los canales de difusión del delito y en tanto apología, explica su impacto en las audiencias vía la formación de actitudes, construcción de representaciones y establecimiento de identidades (Badejo y Oluyemi, 2012). Sin embargo, las representaciones sociales son más bien un contexto histórico en el que convergen una diversidad de símbolos, significados y sentidos orientados a la formación, desarrollo o extinción de un grupo en relación con alguna problemática que les atañe.

Setrata de saberes y conocimientos cotidianos que derivan en discursos, creencias y estereotipos inherentes a algún grupo, o bien, son categorizaciones prototípicas influidas por identidades mayoritarias y minoritarias (Dammert, Salazar, Montt y González, 2010).

A medida que las instituciones públicas se transforman en sociedades anónimas o asociaciones civiles, los recursos naturales y los servicios públicos adquieren una nueva desigualdad que estriba en su administración y por la cual se espera que las capacidades de gestión determinen el desarrollo local, humano y sustentable (Escobar, 2012).

En tal coyuntura, la participación social, política y ciudadana acordes con las políticas públicas son el contrapeso a la discrecionalidad de las autoridades locales, haciendo parecer que es posible un sistema de gestión civil independiente de la acción gubernamental, pero a expensas de las capacidades que el mercado demanda para con usuarios de los servicios públicos quienes son reducidos a meros consumidores de facto (Díaz, 2013).

\section{CONCLUSIONES}

- La principal causa de la inseguridad ciudadana en el distrito de Magdalena del Mar son los robos.

- Los habitantes, ante un acto de seguridad ciudadana acude inmediatamente a llamar a la policía o serenazgo del distrito, aunque un cierto porcentaje prefiere callar debido a posibles represalias.

- Las personas opinan que la situación sobre la seguridad ciudadana en el distrito de Magdalena del Mar en los últimos doce meses no ha tenido cambios significativos referentes a otros años, lo cual demuestra la falta de acción por parte de las autoridades.

- Los habitantes opinan que el principal motivo por el cual se produce la inseguridad ciudadana es la falta de policías y personal de serenazgo en distrito.

- El ejercicio que realiza la policía y serenazgo es poco eficiente, lo cual conlleva a la proliferación de la delincuencia en el distrito. 
- Los habitantes piden más organización social para sentirse más seguro en el distrito.

- Los habitantes califican como regular la eficiencia de los policías ante la presencia de diferentes actos de seguridad ciudadana.

\section{REVISIÓN BIBLIOGRÁFICA}

Araujo, X. (2012). Una revisión básica sobre conceptos y teorías de gobernabilidad. Geoenseñanza, 9, 203-212.

Badejo, A., y Oluyemi, S. (2012). Predisposing factors to youth involvement in electoral violence in Lagos metropolis. Journal of Emerging Trends in Educational Research and Policing Studies. 3, 312-316.

Basombrio, C. (2003). En su tesis "Plan Nacional del Sistema de la Seguridad Ciudadana en lima Perú - 2003".

Bolivar, C., Contreras, J. Jiménez, M. y Chaux, E. (2010). Desentendimiento moral y dinámicas del robo escolar. Revista de Criminología. 52, 243-261 página 14 margen71.

Cuadra, E. (2015). VII Encuesta sobre de percepciones sobre la seguridad ciudadana. Instituto de Estudios Estratégicos y Políticas Públicas. Nicaragua.

Dammert, L., Salazar, F., Montt, C., y González, P. (2010). Crimen e inseguridad. Indicadores para las Américas. Santiago: Flacso-Bid.

Díaz, C. (2013). Nueva gestión pública y gobernanza: desafíos en su implementación. International Journal of Conscience, 8, 177-194.

Escobar, M. (2012). La participación ciudadana, análisis a partir de la transición democrática. Revista Internacional de Investigación en Ciencias Sociales, 8, 119-140. 